\title{
On Some Positivity Properties of the Interquark Potential in QCD
}

\author{
Shmuel Nussinov网 \\ School of Physics and Astronomy \\ Raymond and Beverly Sackler Faculty of Exact Sciences \\ Tel Aviv University \\ 69978 Tel Aviv, Israel \\ and \\ Department of Physics and Astronomy \\ University of South Carolina \\ Columbia, South Carolina 29208, USA
}

\begin{abstract}
We prove that the Fourier transform of the exponential $e^{-\beta V(R)}$ of the static interquark potential in QCD is positive. It has been shown by Eliott Lieb some time ago that this property allows in the same limit of static spin independent potential proving certain mass relation between baryons with different quark flavors.
\end{abstract}

*Electronic mail: nussinov@ccsg.tau.ac.il 
Convex potentials and potentials with positive laplacians have bound states with radial/orbital excitations ordered in certain ways [1] [2]. The convexity of $V(r)$ has been proven some time ago [3].

Here we would like to argue that the Fourier transform of $e^{-\beta V(r)}$ is positive. E. Lieb [4] has shown that this property implies, in particular the inequality (first conjectured in [5]):

$$
M(m m m)+M(m \mu \mu)=2 M(m m \mu)
$$

for baryons made of quarks with the above masses and with spin and flavor (mass) independent pairwise interaction $V(r)$.

Following Wilson we define for any closed path $c$ in an Euclidean four-dimensional Lattice:

$$
W(c)=<\operatorname{tr} U c>
$$

with

$$
U_{c}=\prod_{l=l i n k s \in c} U_{l}
$$

the path ordered product of the $U$ matrices associated with the links of $c$. The Euclidean path integral average is indicated by $<>$. It involves integrating over all $d U_{l}$ with the positive weight:

$$
e^{-\sum_{\text {plaquettes }} \operatorname{tr}\left(U(p)+U^{+}(p)-2\right)} \prod_{i} \operatorname{det} S_{F}^{(i)}(U)
$$

with $\left(S_{F}^{i}(U)\right.$ the fermion propagator in the $U$ background and can be shown to be positive [6], [7], [8]. Consider the special case where $c=c(R, T)$ is a rectangle of "time-direction" height $T$ and width $R$. The static potential between two heavy quarks separated by a distance $R$ is given by

$$
V(R)=\lim _{T-\infty}-\frac{1}{T} \ln W(R, T)
$$

The argument for (4) is well-known.

Let us start at $t=0$ with a $Q \bar{Q}$ state with the quark $Q$ and anti-quark $\bar{Q}$ at $\pm \frac{\vec{R}}{2}$. To maintain gauge invariance we should multiply the state by a Wilson line factor, $\Pi U_{i}$, containing the product of the $U$ 's on $c\left(-\frac{\vec{R}}{2}, \frac{\vec{R}}{2}\right)$, namely on the spatial links along some path connecting $\frac{\vec{R}}{2}$ and $-\frac{\vec{R}}{2}$. The latter is usually chosen to be the straight line of length $R$ connecting $+\frac{\vec{R}}{2}$ and $-\frac{\vec{R}}{2}$. We then let the system propagate for time $T$. The (very) 
massive quarks do then stay at the initial location and hence propagte over the straight "pure time" lines $L_{Q}, L_{\bar{Q}}$ connecting $\left(+\frac{\vec{R}}{2}, 0\right)$ with $\left(-\frac{\vec{R}}{2}, T\right)$ and $\left(+\frac{\vec{R}}{2}, 0\right)$ with $\left(+\frac{\vec{R}}{2}, T\right)$. In the process $Q$ picks up the Wilson factor $U_{L_{1}}$ associated with the upgoing $L_{1}$ and $\bar{Q}$ picks up $U_{L_{2}}^{+}=U_{-L_{2}}$, the conjugate or line-reversed Wilson factor for $L_{2}$. The complete evolution of the system, given by

$$
<\psi_{f Q \bar{Q}}\left|e^{i H T}\right| \psi_{i Q \bar{Q}}>
$$

will then have the four elements of $W(R, T)$ pieced together into one closed loop. (Recall that $<\psi_{f Q \bar{Q}} \mid$ includes the opposite Wilson line (from $-\frac{\vec{R}}{2}$, to $\left.\frac{\vec{R}}{2}\right)$.) This becomes the matrix element of $e^{-H T}$ after Wick rotation to the Euclidean regime. For static quarks this is simply: $e^{-2 M T} e^{-V(R) T}$. It is calculable via the Euclidean langragian path integral leading to

$$
e^{(-V(R)) T} \approx<\operatorname{tr} U(R) T>=<W(R, T)>
$$

where the factor $e^{-2 M T}$ associated with the free propagation of the two heavy quarks is omitted, and equation (5) readily follows.

We can separate $U(c)=U_{L} U_{R}^{+}$into the product of all $U$ 's lying on links to the left (right) of, say the $x=0$ reflection plane and correspondingly separating the $d U_{L} d U_{R}$ integration measure. We can then compare $W(r, T) W(R, T)$ and $W\left(\frac{R+r}{2}, T\right)$. A Schwartz inequality leads to

$$
W(r, T) W(R T) \geq W^{2}\left(\frac{R+r}{2}, T\right)
$$

which using (4) implies the convexity of the potential

$$
V\left(\frac{R+r}{2}\right) \geq \frac{1}{2}[V(R)+V(r)]
$$

Let us next prove the positivity of the Fourier transform (F.T.) of $e^{-T V(r)}$. We will do so by a slight modification of the proof of the positivity of F.T. of certain four-point functions [9]. Let us then consider the following evolution which is a slight modification of that describe above in the proof of equation (4). Let the heavy quarks $Q \bar{Q}$ be produced at $t=0$ and $\vec{r}=0$, and then separate, still at $t=0$, by moving $Q$ to $\vec{r}$ and $\bar{Q}$ to $\vec{r}_{2}$. After pure-timelike propagation to $t=T$ we bring back $Q$ and $\bar{Q}$ to $\vec{r}=0$ and annihilate them there. In this case the Wilson loop factor $W\left(\vec{r}_{1}, \vec{r}_{2}, T\right)$ corresponds to the closed path of fig. 1.

We can separate it into the product of $U$ matrices along the " $\vec{r}_{1}$ like line" (going from $\overline{0}, 0$ to $\vec{r}_{1}, 0$, then to $\vec{r}_{1} T$ and then to $\left.0, T\right)$ and a similar product over the reverse $\vec{r}_{2}$ line" (connecting $\overrightarrow{0}, 0$ to $\vec{r}_{2}, 0$ to $\vec{r}_{1}, T$ to $\overrightarrow{0}, T$ ) 


$$
U=U\left(\vec{r}_{1}\right) U^{\dagger}\left(r_{2}\right)
$$

We are interested in the Fourier transform of $e^{-T V(R)}$. To find the latter courier

$$
L(\vec{P}, T) \equiv \int d \vec{r}_{1} d \vec{r}_{2} e^{i \vec{p}\left(\vec{r}_{1}-\vec{r}_{2}\right)}<W_{c}\left(\vec{r}_{1}, \vec{r}_{2} T\right)>
$$

By exchanging orders of the trace, the $d \vec{r}_{1}, d \vec{r}_{2}$ integration and the $d \mu(U)$ path integral averaging we can write:

$$
\begin{aligned}
L(\vec{P}, T)=\int d \mu(U) \operatorname{tr} & {\left[\left(\int d \vec{r}_{1} U\left(\vec{r}_{1}\right) e^{i \vec{P} \vec{r}_{1}}\right)\right.} \\
& \left.\left(\int d \vec{r}_{2} U\left(\vec{r}_{2}\right) e^{i \vec{P}_{1} \vec{r}_{2}}\right)^{\dagger}\right]
\end{aligned}
$$

which is manifestly positive definite. Next we note that apart from some initial and final small corrections the $Q$ and $\bar{Q}$ were at a fixed distance $\left|\vec{r}_{1}-\vec{r}_{2}\right|$ for all the duration of the evolution. Hence we expect, particularly in the $T \rightarrow \infty$ limit of interest, that 10 ]

$$
W_{c}\left(\vec{r}_{1}, \vec{r}_{2}, T\right) \underset{T \rightarrow \infty}{\approx} E^{-V\left(\vec{r}_{1}-\vec{r}_{2}\right) T}
$$

The positivity of $L(P, T)$ then implies the desired positivity of the Fourier transform of $e^{-T V(r)}$.

The author would like to acknowledge the hospitality of the Department of Physics of the University of Maryland. 


\section{REFERENCES}

[1] G. Feldman and T. Fulton, Nucl. Phys. B154, 441 (1979).

[2] B. Baumgartner, H. Grosse, and A. Martin, Nucl. Phys. B254, 528 (1985); ibid Phys. Lett. B146, 363 (1984).

[3] C. Bachas, Phys. Rev. D 33, 2723 (1986);

E. Seiler, Phys. Rev. D 18, 482 (1978).

[4] E. Lieb, Phys. Rev. Lett. 54, 1987 (1985).

[5] S. Nussinov, Phys. Rev. Lett. 51, 2081 (1983).

[6] D. Weingarten, Phys. Rev. Lett. 51, 1830 (1983).

[7] C. Vafa and E. Witten, Nucl. Phys. B234, 173 (1984).

[8] S. Nussinov and M. Lappert, QED Inequalities submitted to Physics Reports, hep-ph $99 / 11 / 532$.

[9] S. Nussinov, Phys. Rev. Lett. 51, 2081 (1983).

[10] From confinement we expect that $\left\langle W_{c}>\propto e^{-A_{c}}\right.$ with $A_{c}$ the area of the minimal surface spanned by $c$. In the case of fig. 1 this surface clearly does not consist of the rectangular faces 1 and 2 of area $\left|r_{1}\right| T$ and $\left|r_{2}\right| T$ respectively, but rather mainly of face the third face with area $\left|\vec{r}_{1}-\vec{r}_{2}\right| T$, (recall that $\left|r_{1}\right|+\left|r_{2}\right| \geq\left|\vec{r}_{1}-\vec{r}_{2}\right|$ is the triangle inequality).

The finite "end-caps" effect translates here to contribution of the area of the two top/bottom triangles of sides $\vec{r}_{1}, \vec{r}_{2}$ and $\left(\vec{r}_{1}-\vec{r}_{2}\right)$ which are $T$ independent and irrelevant in the $T \rightarrow \infty$ limit of interest. We note however that these contributions proportional to $e^{-r_{1}{ }^{2}\left(\text { or } r_{2}{ }^{2}\right)}$ or to $e^{-M\left(\vec{r}_{1}-\vec{r}_{2} \mid\right.}$ ensure that the $d\left(\vec{r}_{1}+\vec{r}_{2}\right)$ integration in (10) or (11), which does not pertain to the F.T. of $e^{-T V\left(\vec{r}_{1}-\vec{r}_{2}\right)}$, converges. 


\section{FIGURES}

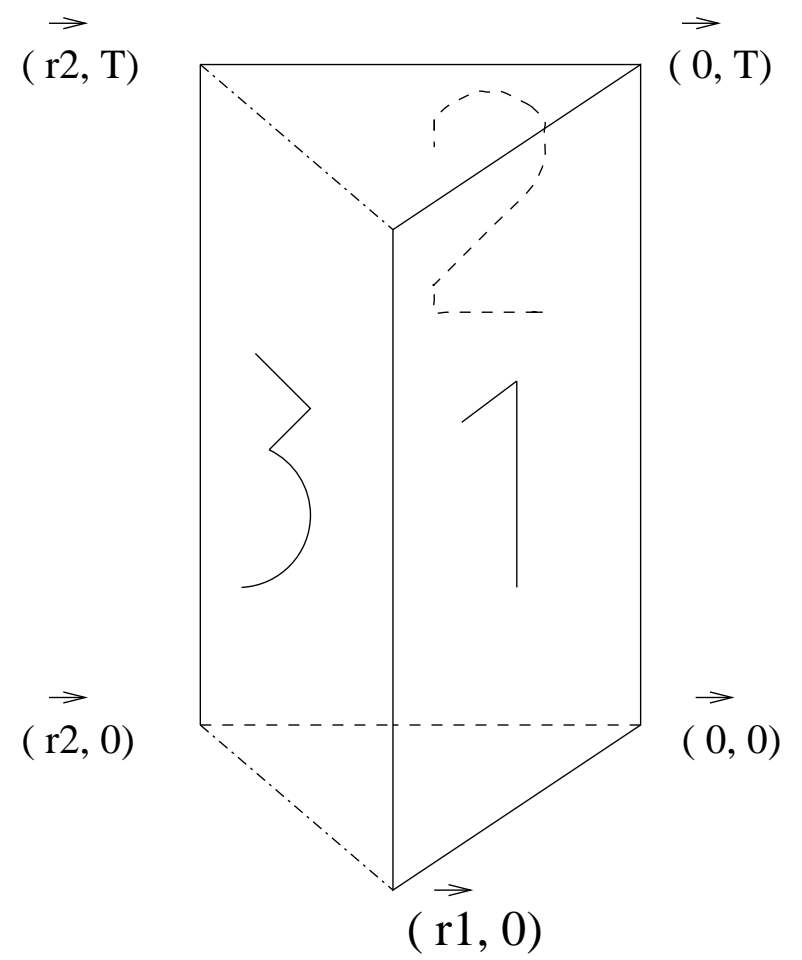

FIG. 1. The location of initial $t=0$ and final $t=T$ heavy quarks and the Wilson paths described by them. 